\title{
Large- $N$ limit of a magnetic impurity in unconventional density waves
}

\author{
Balázs Dóra \\ The Abdus Salam ICTP, Strada Costiera 11, I-34014, Trieste, Italy
}

(Dated: November 20, 2018)

\begin{abstract}
We investigate the effect of unconventional density wave (UDW) condensate on an Anderson impurity using large- $N$ technique at $T=0$. In accordance with previous treatments of a Kondo impurity in pseudogap phases, we find that Kondo effect occurs only in a certain range of parameters. The f-electron density of states reflects the influence of UDW at low energies and around the maximum of the density wave gap. The static spin susceptibility diverges at the critical coupling, indicating the transition from strong to weak coupling. In the dynamic spin susceptibility an additional peak appears showing the presence the UDW gap. Predictions concerning non-linear density of states are made. Our results apply to other unconventional condensates such as d-wave superconductors and d-density waves as well.
\end{abstract}

PACS numbers: 75.30.Fv, 71.45.Lr, 71.10.Hf, 75.20.Hr

\section{INTRODUCTION}

Our understanding of the problem of dilute concentration of magnetic impurities in normal metals benefited a lot from exact solutions, and from reliable approximate methods ${ }^{1}$. Among the latter, the large- $N$ expansion seems to describe successfully the low temperature properties of matter, $N$ denotes the spin degeneracy, and $1 / N$ can be used as an expansion parameter ${ }^{2.3 .4}$. On the other hand, recently considerable attention has been focused on the behaviour of magnetic impurities in pseudogap phases, where the conduction electron density of states varies as a power-law of energy around the Fermi energy 5.6.7.8.9.10.11. The interest on this subject mainly arises due to the behaviour of the pseudogap and superconducting phase of high $T_{c}$ superconductors. The interplay between unconventional condensates and quantum magnetic impurities can reveal the nature of the underlying phase. Using large- $N$ technique, several interesting results were found concerning the transition from magnetic to non-magnetic phases, the quasiparticle density of states etc. It was stated that particle-hole symmetry breaking is necessary to enter into the Kondo regime $\underline{10}$, which condition is naturally satisfied with an infinite- $\mathrm{U}$ Anderson impurity.

Experimentally, from scanning tunneling microscopy studies in $\mathrm{Bi}_{2} \mathrm{Sr}_{2} \mathrm{CaCu}_{2} \mathrm{O}_{8+\delta} 12.13$, a strong variation of the electron density of states was found around the impurity site: beyond the superconducting coherence peaks, new structures were identified indicating the presence of impurity induces bound states. Some of the experiments can be explained by pure potential scattering, but other features call for theoretical works to understand the effect of unconventional condensates on magnetic impurities 14 .

This is why we have chosen to study the interaction between unconventional density waves (UDW) and magnetic impurities. In UDW, the gap in the quasiparticle spectrum vanishes on certain subsets of the Fermi surface, and its average over the Fermi surface is zero 15.16 . This causes the lack of periodic modulation of the spin and charge density. Such states have been proposed to describe the pseudogap phase of high $T_{c}$ superconductors ${ }^{16.17 .18}$, the low temperature phase of $\alpha$-(BEDT-TTF $)_{2} \mathrm{KHg}(\mathrm{SCN})_{4}{ }^{19.20}$, the antiferromagnetic phase in $\mathrm{URu}_{2} \mathrm{Si}_{2}{ }^{21.22}$ and other heavy fermion materials ${ }^{23}$, the charge density wave in $2 \mathrm{H}-\mathrm{TaSe}_{2}{ }^{24}$ and the pseudogap phase in transition metal oxides ${ }^{25}$. Due to the wavevector dependence of the gap, the transition to this phase is metal to metal instead of metal to insulator, as in conventional density waves (with constant energy gap).

The paper is organized as follows: in Sec. II, we study an Anderson impurity embedded in an unconventional density wave in the large- $N$ limit at $T=0$. As opposed to previous treatments ${ }^{8.9}$, we allow for a macroscopic occupation of the $f$ state. From the saddle point equations, the phase diagram is determined, and the effect of magnetic field is discussed. In Sec. III, we turn to the investigation of the properties of the impurity. Its density of states displays the same power-law energy dependence at low energies as that of band electrons, enhances around $\pm \Delta$ and a Kondo peak shows up at positive energies. The presence of these three peaks is in accord with experimental findings in $\mathrm{Bi}_{2} \mathrm{Sr}_{2} \mathrm{CaCu}_{2} \mathrm{O}_{8+\delta} 12.13$. The peculiarities of the conduction electron transport lifetime are explored. The static spin susceptibility signals the transition from the Kondo to the decoupled free moment regime. The dynamic spin susceptibility exhibits the usual Kondo peak, plus an additional peak coming from the divergent peak in the density of states of UDW. Some generalizations to non-linear density of states are made. In spite of the different topology of the Fermi surfaces, our results apply to other phases with power-law density of states like in d-wave superconductors $\stackrel{26}{ }$ or in d-density waves 27 . 


\section{PHASE DIAGRAM}

The Hamiltonian describing an infinite-U Anderson impurity interacting with an unconventional density wave is given by:

$$
\begin{gathered}
H=\sum_{\mathbf{k}, m}^{\prime}\left[\xi(\mathbf{k})\left(a_{\mathbf{k}, m}^{+} a_{\mathbf{k}, m}-a_{\mathbf{k}-\mathbf{Q}, m}^{+} a_{\mathbf{k}-\mathbf{Q}, m}\right)+\Delta(\mathbf{k})\left(a_{\mathbf{k}, m}^{+} a_{\mathbf{k}-\mathbf{Q}, m}+a_{\mathbf{k}-\mathbf{Q}, m}^{+} a_{\mathbf{k}, m}\right)+\right. \\
\left.\frac{V}{\sqrt{N}}\left(\left(a_{\mathbf{k}, m}^{+}+a_{\mathbf{k}-\mathbf{Q}, m}^{+}\right) f_{m} b^{+}+f_{m}^{+}\left(a_{\mathbf{k}, m}+a_{\mathbf{k}-\mathbf{Q}, m}\right) b\right)\right]+E \sum_{m} f_{m}^{+} f_{m},
\end{gathered}
$$

where $a_{\mathbf{k}, m}^{+}$and $a_{\mathbf{k}, m}$ are, respectively, the creation and annihilation operators of an electron of momentum $\mathbf{k}$ and spin $m,-s \leq m \leq s, N=2 s+1$. Similarly, $f_{m}^{+}$and $f_{m}$ creates and annihilates an electron on the localized $E$ level, $b^{+}$and $b$ are the slave boson operators, responsible for the hole states 2.3 . In a sum with prime, $k_{x}$ runs from 0 to $2 k_{F}$ ( $k_{F}$ is the Fermi wavenumber), $\mathbf{Q}=\left(2 k_{F}, \pi / b, \pi / c\right)$ is the best nesting vector. $\Delta(\mathbf{k})=\Delta \sin \left(b k_{y}\right)$ is the unconventional density wave order parameter, and is taken to be real for simplicity. A gap with $\cos \left(b k_{y}\right)$, or $k_{y} \rightarrow k_{z}$ replacement would yield to the same results. Our system is based on an orthogonal lattice, with lattice constants $a, b, c$ toward direction $x, y, z$. The system is anisotropic, the quasi-one-dimensional direction is the $x$ axis. The $a$ electron system possesses a density of states, which varies linearly with energy around the Fermi energy: $N(\omega) / N_{0}=|\omega| / \Delta$ for $|\omega| \ll \Delta, N_{0}$ is the normal state density of states per spin at the Fermi energy ${ }^{15}$. The linearized kinetic-energy spectrum is $\xi(\mathbf{k})=v_{F}\left(k_{x}-k_{F}\right)$, and the dispersion in the other directions can be neglected for practical purposes. By introducing the spinor

$$
\Psi(\mathbf{k}, m, \tau)=\left(\begin{array}{c}
a_{\mathbf{k}, m}(\tau) \\
a_{\mathbf{k}-\mathbf{Q}, m}(\tau)
\end{array}\right)
$$

the single-particle thermal Green's function of the $a$ electrons without hybridization is obtained from Eq. (1) as

$$
G\left(\mathbf{k}, i \omega_{n}\right)=-\int_{0}^{\beta} d \tau\left\langle T_{\tau} \Psi(\mathbf{k}, m, \tau) \Psi^{+}(\mathbf{k}, m, 0)\right\rangle_{H} e^{i \omega_{n} \tau}=\left[i \omega_{n}-\xi(\mathbf{k}) \rho_{3}-\rho_{1} \Delta(\mathbf{k})\right]^{-1},
$$

where $\omega_{n}$ is the fermionic Matsubara frequency, $\rho_{i}$ 's $(i=1,2,3)$ are the usual Pauli matrices acting on momentum space.

The Hamiltonian should be restricted to the subspace

$$
\sum_{m} f_{m}^{+} f_{m}+b^{+} b=Q
$$

where in the true large- $N$ limit $Q$ grows extensively with $N$, i.e. $Q=N q, 0<q<1$. In the original formulation of the model, $Q=1$, but in order to preserve a macroscopic occupation of the $f$ state, the above generalization was found to be useful ${ }^{\underline{3}}$. It has been argued that the properties of the spin- $\frac{1}{2}$ Anderson model are best represented by $q=1 / 2$ in the large- $N$ limit $^{2}$. Within the mean-field approximation, the slave-boson operators are replaced by their expectation value, $b_{0}=\langle b\rangle / \sqrt{N}$, and the constraint is satisfied by introducing a Lagrange multiplier $\lambda$ :

$$
\begin{gathered}
H=\sum_{\mathbf{k}, m}^{\prime}\left[\xi(\mathbf{k})\left(a_{\mathbf{k}, m}^{+} a_{\mathbf{k}, m}-a_{\mathbf{k}-\mathbf{Q}, m}^{+} a_{\mathbf{k}-\mathbf{Q}, m}\right)+\Delta(\mathbf{k})\left(a_{\mathbf{k}, m}^{+} a_{\mathbf{k}-\mathbf{Q}, m}+a_{\mathbf{k}-\mathbf{Q}, m}^{+} a_{\mathbf{k}, m}\right)+\right. \\
\left.V b_{0}\left(\left(a_{\mathbf{k}, m}^{+}+a_{\mathbf{k}-\mathbf{Q}, m}^{+}\right) f_{m}+f_{m}^{+}\left(a_{\mathbf{k}, m}+a_{\mathbf{k}-\mathbf{Q}, m}\right)\right)\right]+(E+\lambda) \sum_{m} f_{m}^{+} f_{m}+N \lambda\left(b_{0}^{2}-q\right),
\end{gathered}
$$

It has been shown ${ }^{3}$ that the slave boson mean-field approximation produces the correct low energy physics of the conventional Anderson impurity model in the entire parameter range at $T=0$. The value of $\lambda$ and $b_{0}$ is determined by minimizing the free energy of the system with respect to them ${ }^{8.9}$. As a result, the saddle point equations at $T=0$ are given by:

$$
\begin{gathered}
b_{0}^{2}=q-\frac{1}{2}+\frac{1}{\pi} \int_{0}^{\infty} \frac{E+\lambda}{x^{2}(1+\tilde{\Gamma} \alpha(x))^{2}+(E+\lambda)^{2}} d x \\
b_{0} \lambda=\frac{b_{0}}{\pi} \int_{0}^{W} \frac{x^{2}(1+\tilde{\Gamma} \alpha(x)) \alpha(x) \Gamma}{x^{2}(1+\tilde{\Gamma} \alpha(x))^{2}+(E+\lambda)^{2}} d x
\end{gathered}
$$


where $\alpha(x)=2 K\left(1 / \sqrt{1+(x / \Delta)^{2}}\right) / \sqrt{\Delta^{2}+x^{2}} \pi$ for $x<W, 0$ otherwise, $K(z)$ is the complete elliptic integral of the first kind. The case of a magnetic impurity embedded in a normal metallic host can be studied with $\alpha(x)=1 /|x|$. $W=v_{F} k_{F}$ is half of the bandwidth, $\Gamma=V^{2} \pi N_{0}, \tilde{\Gamma}=\Gamma b_{0}^{2}$. The critical value of $E$ is obtained as

$$
E_{c}=-\frac{\Gamma}{\pi} \ln \left(\frac{4 W}{\Delta}\right)
$$

in the weak coupling limit $(W \gg \Delta)$. Below this value, only the trivial solution of the saddle point equations exist, namely $b_{0}=0$ and $\lambda=-E$, and no Kondo effect occurs. To study the more general case, when the density of states varies as a power-law of energy, one has to assume $\Delta(\mathbf{k})=\Delta\left|\sin \left(b k_{y}\right)\right|^{r} \operatorname{sign}\left(\sin \left(b k_{y}\right)\right), 0<r<\infty$. The sign function assures the vanishing average of the gap over the Fermi surface, and the $r$ exponent results in a density of states as $N(\omega) / N_{0}=|\omega / \Delta|^{1 / r} \Gamma(1 / 2 r) / \sqrt{\pi} r \Gamma(1+r / 2 r)$ for $|\omega / \Delta|^{1 / r} \ll 1$, and $\Gamma(x)$ is the complete Gamma function. With this, the above condition is modified as

$$
E_{c}=-\frac{\Gamma}{\pi} \ln \left(\frac{2^{r+1} W}{\Delta}\right)
$$

The higher the value of $r$, the lower the critical $f$ level energy, and by letting $r \rightarrow \infty$, the case of a normal metal can be reached with a constant density of states, when $E_{c} \rightarrow-\infty$, which means, that Kondo effect is always present at $T=0$. The saddle point equations have been solved numerically, and the results are shown in Figs. 1 and 2 for $q=1 / 2$ and $q=1 / 6$. For comparison, we also show the results assuming a normal metallic host. As $\Gamma$ decreases, $b_{0}$ increases much sharper, and reaches its maximum value $\sqrt{q}$ rapidly. In spite of the different constraints $(q=1 / 2$ and $1 / 6)$, the similarity between the figures is striking, indicating that the results hardly depend on the filling factors. As to $\lambda$, it equals to $|E|$ below its critical value, and approaches 0 as the $f$ level energy is further increased.

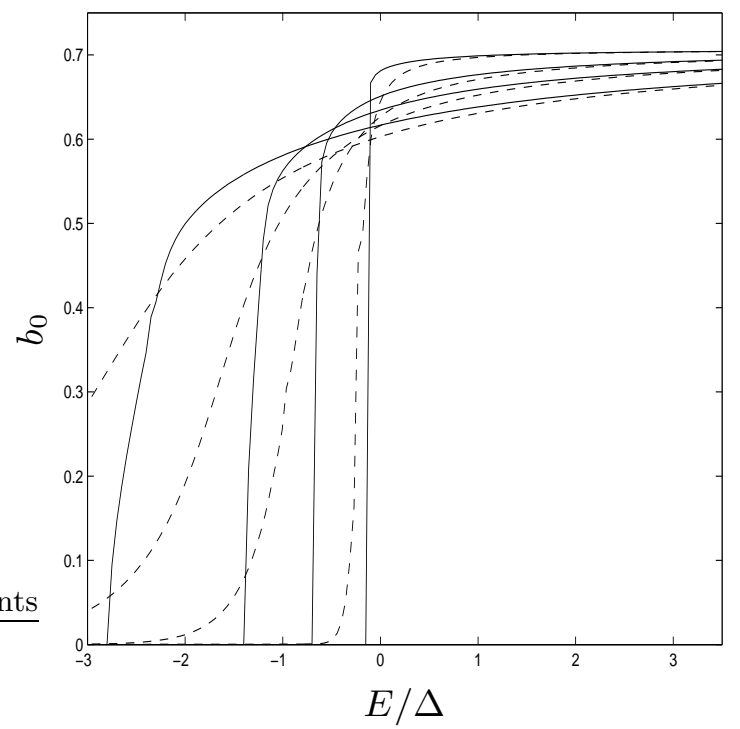

FIG. 1: The $E$ dependence of the order parameter (the expectation value of the slave-boson operator) is plotted for $q=1 / 2$, $W=20 \Delta, \Gamma / \Delta=0.1,0.5,1$ and 2 from right to left (solid line), while the dashed line represents the behaviour of an Anderson impurity embedded in a normal metallic host.

The effect of magnetic field can readily be incorporated into the theory by adding the Zeeman term to the Hamiltonian:

$$
H_{Z e e m a n}=-h \sum_{m} t_{m} f_{m}^{+} f_{m}
$$

where the appropriate choice of $t_{m}$ can represent the two extreme limits 28 : (1) the straightforward generalization of the SU(2) model, where $t_{m}=m / N$ and the magnetic field completely lifts the impurity degeneracy, or (2), when $t_{m}=\operatorname{sign}(m)$, where two $N / 2$-fold degenerate levels are introduced by the field. For large fields, the critical $f$ level 


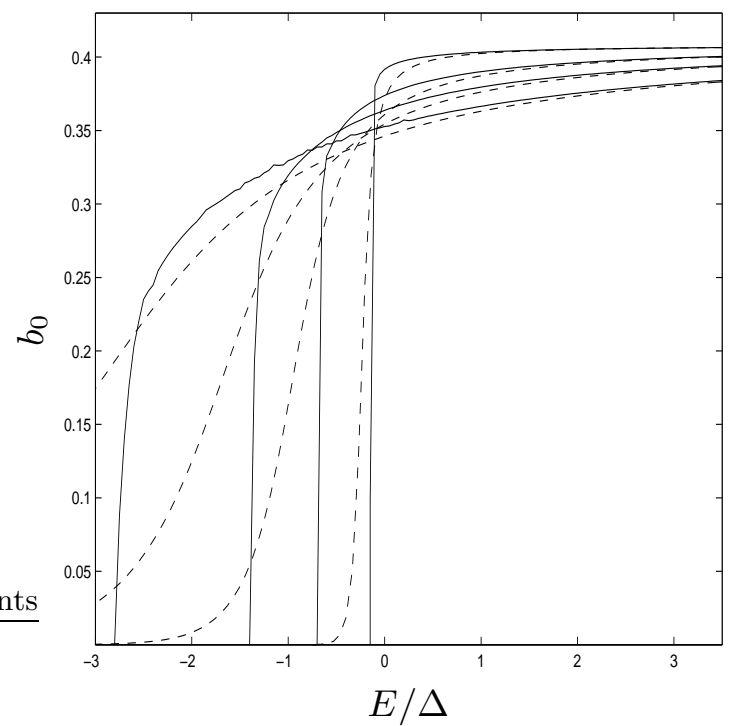

FIG. 2: The $E$ dependence of the order parameter is shown for $q=1 / 6, W=20 \Delta, \Gamma / \Delta=0.1,0.5,1$ and 2 from right to left (solid line), while the dashed line represents the behaviour of an Anderson impurity embedded in a normal metallic host.

energy is obtained as

$$
E_{c} \approx E_{c 0}+|h|\left\{\begin{array}{cc}
\left(\frac{1}{2}-q\right) & \text { for case (1) } \\
\operatorname{sign}\left(\frac{1}{2}-q\right) & \text { for case (2) }
\end{array}\right.
$$

where $E_{c 0}$ is the critical $f$ level energy without magnetic field. For $q \leq 1 / 2$, which is thought to be the physically motivated case, an applied magnetic field enhances the critical $E$, hence destroys the Kondo region. This follows naturally from the fact, that we have $N / 2$ states below $E$ generated by the magnetic field, so the highest occupied state at $T=0$ is below the actual $E$. On the other hand, for $q \geq 1 / 2$, by the use a magnetic field, the system can be driven back into the strong coupling regime, but the physical realization of such a situation (i.e. $q \geq 1 / 2$ ) seems to be doubtful. Here, the highest occupied state generated by the magnetic field is above $E$, which makes the hybridization possible again.

\section{DENSITY OF STATES, SPIN SUSCEPTIBILITY}

The Green's function of the $f$ electrons reads as

$$
G_{f}\left(i \omega_{n}\right)=\frac{1}{i \omega_{n}-E-\lambda+i \omega_{n} \tilde{\Gamma} \alpha\left(\omega_{n}\right)}
$$

where $\alpha(x)$ was defined below Eq. 7 The self energy of the $f$ electrons along the real frequency axis is obtained as

$$
\Sigma_{f}(\omega+i \delta)=-\frac{2 \tilde{\Gamma}}{\pi}\left[\Theta(\Delta-|\omega|)\left(\frac{\omega}{\Delta} K\left(\sqrt{1-\left(\frac{\omega}{\Delta}\right)^{2}}\right)+i\left|\frac{\omega}{\Delta}\right| K\left(\frac{\omega}{\Delta}\right)\right)+\Theta(|\omega|-\Delta) i K\left(\frac{\Delta}{\omega}\right)\right],
$$

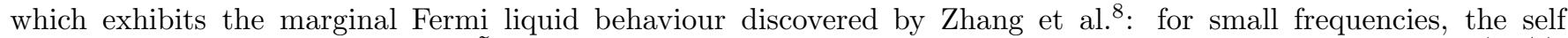
energy varies as $\Sigma_{f}(\omega \ll \Delta)=-\tilde{\Gamma}(2 \omega \ln (4 \Delta /|\omega|) / \pi+i|\omega|) / \Delta$. For $r \neq 1$ exponent, $\operatorname{Re} \Sigma_{f} \sim \operatorname{sign}(\omega)|\omega|^{\min (1,1 / r)}$, $\operatorname{Im} \Sigma_{f} \sim|\omega|^{1 / r}$. In general, the imaginary part of the self energy is directly proportional to the $a$ electron density of states. From the self energy, the real $f$ electron density of states, which is the imaginary part of the Fourier transform of $-\left\langle\mathrm{T}_{\tau} b^{+}(\tau) f_{m}(\tau) f_{m}^{+}(0) b(0)\right\rangle^{3}$, reads as

$$
\rho_{f}(\omega)=-\frac{b_{0}^{2}}{\pi} \frac{\operatorname{Im} \Sigma_{f}(\omega)}{\left(\omega-E-\lambda-\operatorname{Re} \Sigma_{f}(\omega)\right)^{2}+\left(\operatorname{Im} \Sigma_{f}(\omega)\right)^{2}},
$$


which has the same low energy behaviour as the $a$ electron density of states, namely around the Fermi energy $\rho_{f}(\omega) \sim|\omega / \Delta|^{1 / r}$. Also around $\pm \Delta$, it is expected to increase sharply due to the divergence of the UDW density of states, but the divergent peak is suppressed since the denominator in Eq. 14] also diverges, as can be seen in Fig. [3] In fact, $\rho_{f}( \pm \Delta)=0$, which is invisible in Fig. 3 due to its scale. The same phenomenon was discussed in charge density waves in the presence of a non-magnetic impurity ${ }^{29}$. Above $E_{c}$, the large, asymmetric Kondo peak appears, and moves to higher frequencies, even above $\Delta$, as $E$ is further increased.

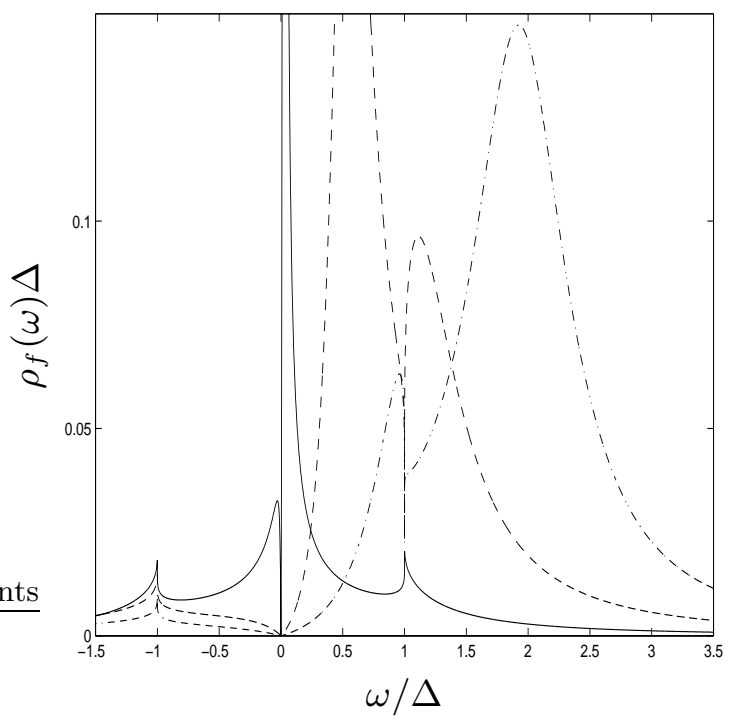

FIG. 3: The $f$ electron density of states is shown for $q=1 / 4$ for $E$ slightly above $E_{c}$ (solid line), $E=0$ (dashed line) and $E=\left|E_{c}\right|$ (dashed-dotted line).

The $T$-matrix of the conduction $(a)$ electrons is obtained as

$$
T(\omega+i \delta)=\frac{V^{2} b_{0}^{2}}{\omega-E-\lambda-\Sigma_{f}(\omega)},
$$

whose poles determine the impurity bound states or resonances 17 . By closely examining the denominator of Eq. 15] we find resonances at the $\Omega_{K}-i \gamma, \Omega_{K}$ can be identified with the Kondo temperature ${ }^{2.14}$, as was done in similar treatments of the large- $N$ limit of the Kondo model ${ }^{7.8 .10}$, and $\gamma$ is the lifetime broadening of the $f$ leve ${ }^{14}$, and is

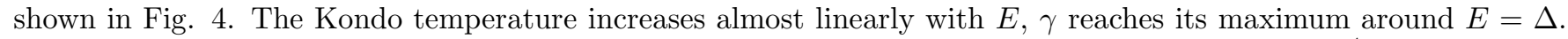
For large $E$, the resonance appears at $E-i q \Gamma$, identically to the case of a normal conduction band ${ }^{4}$. Alternatively, one can define the Kondo energy scale $e^{2.4}$ as $\sqrt{\Omega_{K}^{2}+\gamma^{2}}$, but it hardly differs from $\Omega_{K}$.

The imaginary part of the $T$-matrix, which gives the inverse transport lifetime of the conduction electrons, is proportional to the real $f$ electron density of states, hence for small energies $\tau \sim|\omega|^{-1}$. Its detection is a very subtle issue, since the number of states having this behaviour is $N(\omega) \sim|\omega|$, hence $N(\omega) \tau \sim$ const. The same cancellation is found for the $r \neq 1$ case. Also $\tau$ is expected to be enhanced around $\pm \Delta$ as $\ln (2 \sqrt{2} / \sqrt{|1-| \omega / \Delta||})$. The number of possible states diverges in the same manner as $\tau$, resulting in $N(\omega) \tau \sim \ln ^{2} \sqrt{|1-| \omega / \Delta||}$. For non-linear density of states, similarly to the case of $r=1$, both $\tau$ and $N(\omega)$ diverges around the gap maximum, which gives $N(\omega) \tau \sim \ln ^{2}|1-| \omega / \Delta \|^{1 / 2 \sqrt{r}}$. This behaviour could be checked by experiments, although its detection is not an easy task, because such excitation are suppressed at low temperatures. With the help of the $T$-matrix, the local UDW density of states can be determined at the impurity site:

$$
\rho_{a}(\omega)=\frac{N_{0} \rho_{f}(\omega)(\omega-E-\lambda)^{2} \pi}{\tilde{\Gamma} b_{0}^{2}}
$$

and is shown in Fig. 5 Slightly above $E_{c}$, a sharp resonance at $\Omega_{K}$ modifies the density of states. By increasing $E$, the Kondo peak is broadened, and the new zero at $\omega=E+\lambda$ can completely suppress the peak at $\omega=\Delta$.

Using Eq. 14 the low temperature specific heat is obtained as

$$
C(T \rightarrow 0)=\frac{18 \zeta(3) \tilde{\Gamma}}{\Delta \pi(E+\lambda)^{2}} T^{2}
$$




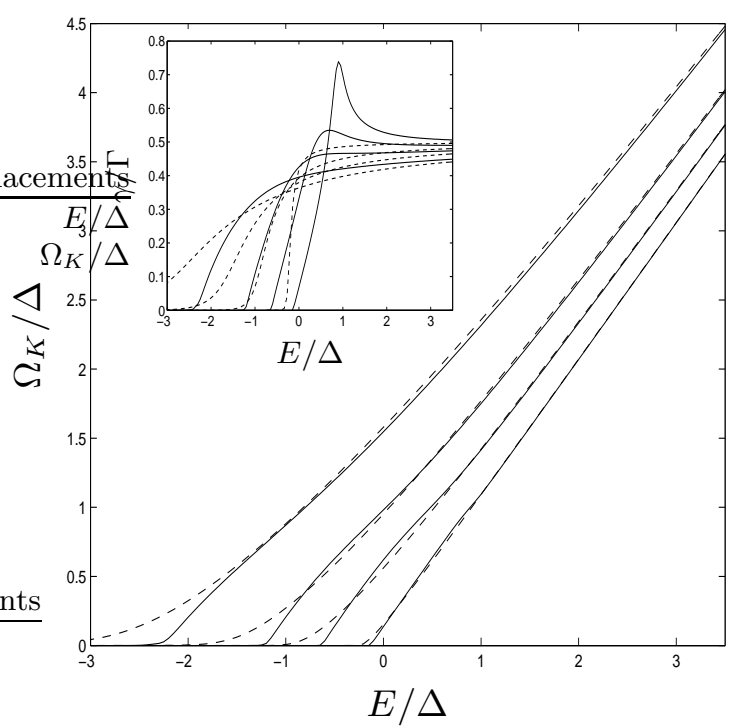

FIG. 4: The Kondo scale, $\Omega_{K}$ is plotted for $q=1 / 2, \Gamma / \Delta=0.1,0.5,1$ and 2 from right to left (solid line). The dashed line represents the behaviour of a normal metallic host. Note that $\Omega_{K}$ never vanishes in this case. The inset shows the lifetime broadening, $\gamma$, together with normal host results.

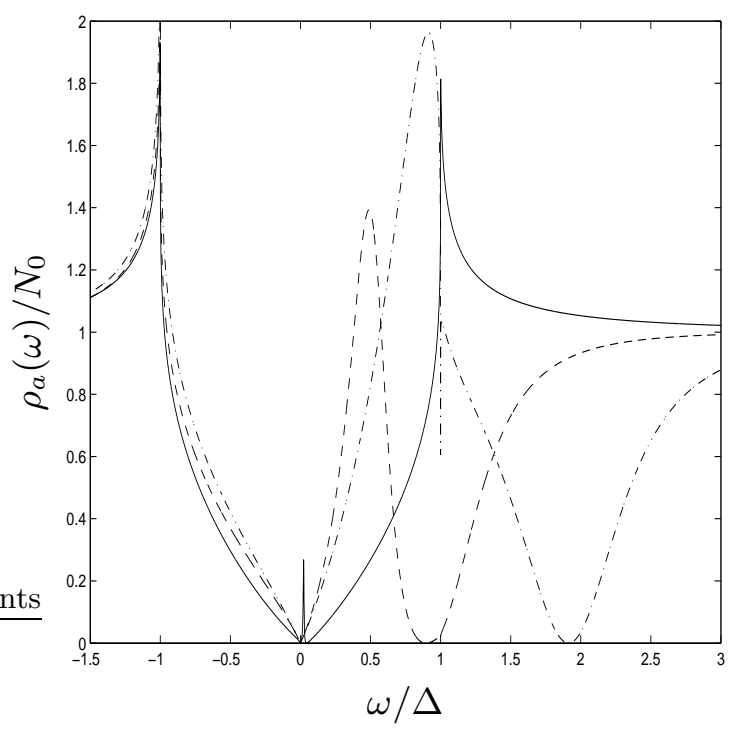

FIG. 5: The local density of states (at the impurity site) of UDW is plotted for $q=1 / 4$ for $E$ slightly above $E_{c}$ (solid line), $E=0$ (dashed line) and $E=\left|E_{c}\right|$ (dashed-dotted line).

which has the same temperature dependence as that of the density wave. For a non-linear density of states, the specific heat varies as $C(T) \sim T^{(1+r) / r}$. Also from the behaviour of $\rho_{f}(\omega)$, the low temperature behaviour of the resistivity can be calculated ${ }^{30}$, and is obtained as $R(T)-R(0) \sim-T^{2} \ln ^{2}(T)$. For general $r \neq 1$ exponents, it decreases as $-T^{\min (2,2 / r)}$.

The impurity spin susceptibility is calculated from

$$
\chi\left(i \omega_{m}\right)=-\mu_{e f f}^{2} T \sum_{n} G_{f}\left(i \omega_{n}+i \omega_{m}\right) G_{f}\left(i \omega_{n}\right),
$$

whose static limit at $T=0$ reads as

$$
\chi(0)=\mu_{e f f}^{2}\left(\frac{b_{0}^{2}-q+\frac{1}{2}}{E+\lambda}-\frac{2}{\pi} \int_{0}^{\infty} \frac{(E+\lambda)^{2}}{\left(x^{2}(1+\tilde{\Gamma} \alpha(x))^{2}+(E+\lambda)^{2}\right)^{2}} d x\right),
$$

which is shown in Fig. [6 together with the corresponding results in a normal metallic host. The susceptibility diverges 
at $E_{c}$, indicating the transition from the mixed valence to the decoupled free spin region, since in the latter region, it behaves as $\chi(T)=\mu_{e f f}^{2} q(1-q) / T$.

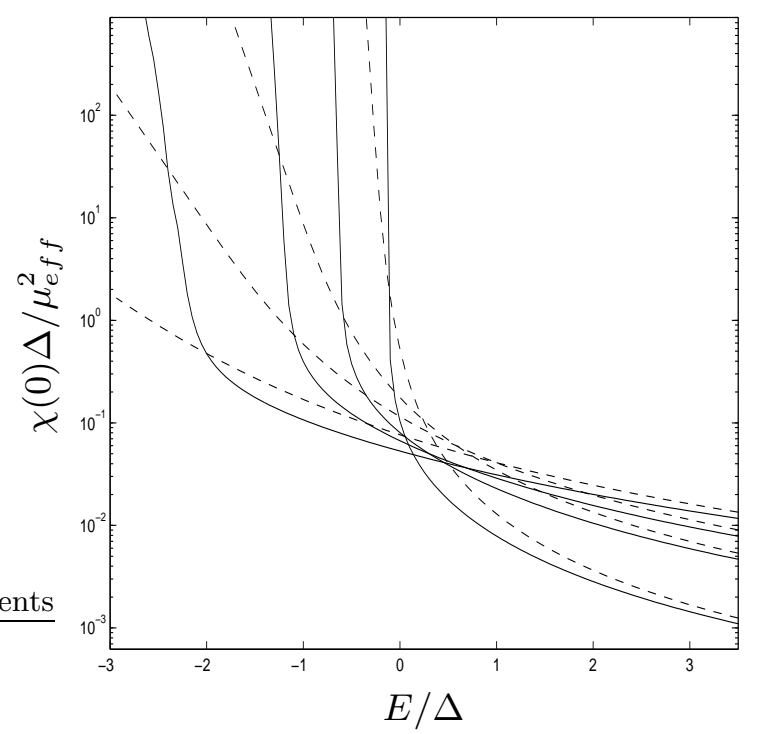

FIG. 6: The $E$ dependence of static spin susceptibility is shown on a semilogarithmic scale for $q=1 / 2, W=20 \Delta, \Gamma / \Delta=0.1$, 0.5, 1 and 2 from right to left (solid line), while the dashed line accounts for the susceptibility of an Anderson impurity embedded in a normal metallic host.

The imaginary part of the impurity spin susceptibility should be readily accessible by neutron scattering experiments, and is evaluated at $T=0$ as

$$
\operatorname{Im} \chi(\omega)=\frac{\mu_{e f f}^{2} \pi}{b_{0}^{4}} \int_{0}^{\omega} \rho_{f}(x) \rho_{f}(x-\omega) d x .
$$

For small frequencies, it behaves as $\operatorname{Im} \chi(\omega)=\tilde{\Gamma}^{2} \omega^{3} / 6 \pi(E+\lambda)^{4} \Delta^{2}$, while for non-linear density of states, it increases as $\omega^{1+2 / r}$. Beyond the usual Kondo peak occurring around $\Omega_{K}$, an additional sharp peak shows up at $\Delta+\Omega_{K}$ corresponding to excitations around the UDW gap. As $E$ increases, the latter becomes dominant, but its sharpness is smeared, as can be seen in Fig. 7 In a pure UDW, only one single peak is expected in $\operatorname{Im} \chi(\omega)$ located at $2 \Delta$, so the presence or absence of the extra two peaks can identify the presence or absence of magnetic impurities.

\section{CONCLUSION}

In summary we have studied the screening of a magnetic impurity in unconventional density waves. We considered an infinite-U Anderson impurity in the large- $N$ limit, when the occupation of the $f$ level increases extensively with $N$. Kondo effect only occurs, if the $f$ level energy or the hybridization matrix element exceeds a certain value, and the result are almost independent of the chosen value of the $f$ level occupation. The impurity density of states exhibits the UDW coherence peaks at $\pm \Delta$, and the usual Kondo peak. The conduction electron density of states at the impurity site displays similar behaviour. The transport lifetime of the conduction electrons is determined from the $T$-matrix, and is found to diverge at $\omega=0$, and $\pm \Delta$. The former is suppressed due to the small number of states possessing this kind of behaviour. The static impurity spin susceptibility diverges at the critical $f$ level energy, where the transition from Kondo to weak-coupling takes place. The dynamic spin susceptibility is enhanced at the Kondo energy, but an additional sharp resonance is noticed belonging to excitations from the gap maximum of UDW states to the Kondo peak. These features can help to identify the magnetic or non-magnetic nature of impurities in pseudogap phases. 


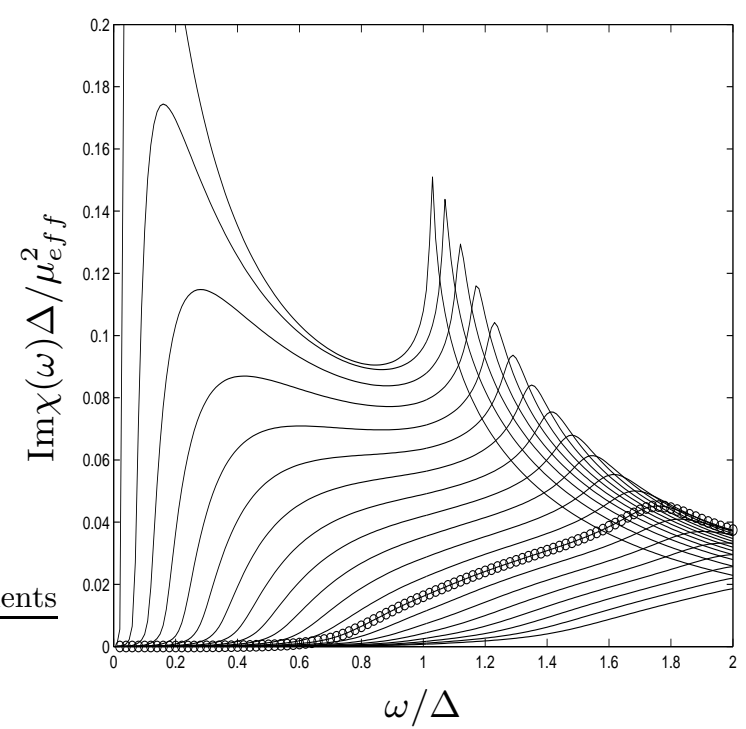

FIG. 7: The evolution of the dynamic spin susceptibility for various values of $E$ (from $E=-1.25 \Delta$ by $0.1 \Delta$ steps from left to right) is shown for $q=1 / 6, W=20 \Delta, \Gamma / \Delta=1$. Two distinct peaks show up above $E_{c}$, and merge as one passes into the Kondo regime with increasing $E$. The circles denote $E \approx 0$.

\section{Acknowledgments}

We are indebted to Gergely Zaránd, Piers Coleman and Boris Narozhny for useful discussions.

1 A. C. Hewson, The Kondo Problem to Heavy Fermions (Cambridge University Press, Cambridge, Great Britain, 1993).

${ }^{2}$ N. E. Bickers, Rev. Mod. Phys. 59, 845 (1987).

${ }^{3}$ P. Coleman, Phys. Rev. B 35, 5072 (1987).

${ }^{4}$ D. M. Newns and N. Read, Adv. Phys. 36, 799 (1987).

${ }^{5}$ D. Withoff and E. Fradkin, Phys. Rev. Lett. 64, 1835 (1990).

${ }^{6}$ L. S. Borkowski and P. J. Hirschfeld, J. Low Temp. Phys. 96, 185 (1994).

7 C. R. Cassanello and E. Fradkin, Phys. Rev. B 53, 15079 (1996).

8 G.-M. Zhang, H. Hu, and L. Yu, Phys. Rev. Lett 86, 704 (2001).

${ }^{9}$ J.-X. Zhu and C. S. Ting, Phys. Rev. B 63, 020506 (2000).

10 A. Polkovnikov, Phys. Rev. B 65, 064503 (2002).

${ }^{11}$ C. Gonzalez-Buxton and K. Ingersent, Phys. Rev. B 57, 14254 (1998).

12 E. W. Hudson, K. M. Lang, V. Madhavan, S. H. Pan, H. Eisaki, S. Uchida, and J. C. Davis, Nature (London) 411, 920 (2001).

13 S. H. Pan, E. W. Hudson, K. M. Lang, H. Eisaki, S. Uchida, and J. C. Davis, Nature (London) 403, 746 (2000).

14 A. Polkovnikov, S. Sachdev, and M. Vojta, Phys. Rev. Lett. 86, 296 (2001).

15 B. Dóra and A. Virosztek, Eur. Phys. J. B 22, 167 (2001).

${ }^{16}$ S. Chakravarty, R. B. Laughlin, D. K. Morr, and C. Nayak, Phys. Rev. B 63, 094503 (2001).

17 C. P. Moca, Phys. Rev. B 66, 132509 (2002).

18 L. Benfatto, S. Caprara, and C. Di Castro, Eur. Phys. J. B 17, 95 (2000).

19 B. Dóra, A. Virosztek, and K. Maki, Phys. Rev. B 64, 041101(R) (2001).

20 B. Dóra, A. Virosztek, and K. Maki, Phys. Rev. B 65, 155119 (2002).

${ }^{21}$ H. Ikeda and Y. Ohashi, Phys. Rev. Lett. 81, 3723 (1998).

22 A. Virosztek, K. Maki, and B. Dóra, Int. J. Mod. Phys. B 16, 1667 (2002).

${ }^{23}$ Zs. Gulácsi and M. Gulácsi, Phys. Rev. B 36, 699 (1987).

${ }^{24}$ A. H. Castro-Neto, Phys. Rev. Lett. 86, 4382 (2001).

${ }^{25}$ D. F. Schroeter and S. Doniach, Phys. Rev. B 66, 075120 (2002).

${ }^{26}$ K. Maki, in Lectures on the Physics of Highly Correlated Electron Systems, edited by F. Mancini (AIP Conference Proceedings 438, Woodbury, 1998), p. 83.

${ }^{27}$ C. Nayak, Phys. Rev. B 62, 4880 (2000).

${ }^{28}$ D. Withoff and E. Fradkin, Phys. Rev. B 34, 8172 (1986).

29 I. Tüttő and A. Zawadowski, Phys. Rev. B 32, 2449 (1985). 
${ }^{30}$ P. Coleman, in Lectures on the Physics of Highly Correlated Electron Systems, edited by F. Mancini (AIP Conference Proceedings 629, Woodbury, 2001), p. 79. 